Revue d'histoire des chemins de fer

\section{Revue d'histoire des chemins de fer}

$41 \mid 2010$

Approvisionnement ferroviaire et pratiques alimentaires des citadins

\title{
Les sources archivistiques du thème chemins de fer et alimentation
}

Archival sources on the relationship between railways and food supply

\section{Laurence Bour}

\section{(2) OpenEdition}

\section{Journals}

Édition électronique

URL : https://journals.openedition.org/rhcf/1141

DOI : 10.4000/rhcf.1141

\section{Éditeur}

Rails \& histoire

\section{Édition imprimée}

Date de publication : 30 avril 2010

Pagination : 21-38

ISSN : 0996-9403

\section{Référence électronique}

Laurence Bour, "Les sources archivistiques du thème chemins de fer et alimentation », Revue

d'histoire des chemins de fer [En ligne], 41 | 2010, mis en ligne le 30 avril 2012, consulté le 22 avril 2022. URL : http://journals.openedition.org/rhcf/1141 ; DOI : https://doi.org/10.4000/rhcf.1141 
Laurence BOUR

\section{Les sources archivistiques du thème chemins de fer et alimentation}

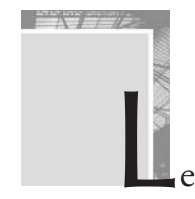

Centre des archives historiques de la SNCF, partie intégrante du Service des Archives et de la Documentation, a ouvert ses portes au Mans en 1995. Depuis lors, il s'efforce de remplir les missions suivantes : collecte des archives historiques au niveau national, tri, classement, élaboration d'instruments de recherche et, bien sûr, communication des archives. Aujourd'hui vingt-cinq kilomètres linéaires de rayonnages sont occupés, sur les quarantequatre kilomètres dont nous disposons. Depuis 2006, nous enregistrons un fort accroissement du volume annuel des versements, de l'ordre de trois à quatre kilomètres par an. Ces versements nous permettent bien évidemment d'enrichir nos fonds.

Le Centre des archives historiques du Mans a la particularité de conserver également un très riche fonds d'imprimés, d'ouvrages et de publications sérielles sur le thème du chemin de fer et des transports en général. Nous ferons donc de fréquentes références à nos fonds documentaires, hérités de la bibliothèque de La Vie du rail et du Centre de documentation de la SNCF, dont l'existence remonte à la création de l'entreprise. Des générations de documentalistes ont fourni un remarquable travail d'analyse d'ouvrages et d'articles de revues, tant en langue française qu'étrangère. 
Notre approche sera toutefois avant tout française et centrée sur les sources présentes au Centre du Mans, l'exposé des sources complémentaires restant à réaliser.

Après avoir abordé les sources permettant une approche par les produits, nous nous intéresserons à l'action des chemins de fer pour limiter les pertes et les avaries par le biais des emballages et des méthodes de manutention. La question primordiale du ravitaillement de la population sera ensuite évoquée, à travers les gares-marchés. Deux autres aspects du sujet sont la nécessité pour le chemin de fer d'assurer la conservation des denrées périssables par le biais d'installations frigorifiques et de mettre en service un matériel de transport adapté. La volonté d'améliorer les conditions de chargement et de déchargement et de réduire ainsi les coûts de manutention conduit au développement de l'utilisation de la palette, puis du conteneur, et à la mécanisation de la manutention. Détaillant la chaîne qui relie le producteur au consommateur, nous consacrerons un paragraphe aux embranchements particuliers. Un autre élément de cette chaîne est la problématique de la réduction des frais afférents aux transports terminaux. Nous aborderons, en dernier lieu, l'état de nos sources sur le thème de la restauration ferroviaire.

\section{Pour une approche par les produits}

Attirés par les perspectives de trafic et donc de recettes, les réseaux de chemins de fer ont mené une active propagande en faveur du développement de l'agriculture. Les «services agricoles » du PO, du Midi, du PLM et de l'État ont multiplié les publications destinées à promouvoir tel ou tel produit, le plus souvent en lien avec des congrès professionnels ou des missions d'études. Une vingtaine de ces ouvrages sont conservés au Mans dans nos fonds documentaires $^{1}$.

Pour une approche par produits, il faudrait aussi exploiter les brochures commerciales, autres que les premières citées, versées au Mans par la direction Grandes Lignes de la SNCF${ }^{2}$. Éditées tant par les anciens réseaux, le PLM notamment, que par la société nationale, estampillées par le Service commercial de la SNCF, elles n'ont pas été analysées, mais simplement classées par origine (réseaux, société nationale), puis dans l'ordre du numéro attribué par l'estampille. Une étude faciliterait leur recensement.

1- Voir dans ce volume l'étude de Georges Ribeill, «Les “Services agricoles” des grands réseaux : de grands moyens pour quelle efficacité ?», p. 61.

2- Versement 470LM. 
Certaines des brochures, signées d'ingénieurs agronomes, dispensent des conseils de culture ${ }^{3}$. D'autres, plus spécifiquement consacrées au transport sous l'angle commercial, donnent des informations touchant aux horaires et aux durées des trajets et à l'organisation du transport proprement dite ${ }^{4}$. Souvent accompagnées de cartes, elles ventent la rapidité de l'acheminement, la fraîcheur des produits à l'arrivée.

Ces brochures sont également une source pour l'étude de l'évolution de la tarification des différents produits. Elles sont cependant moins riches que les fiches présentes dans le versement 44LM qui, pour l'essentiel, portent sur les modifications de tarification évoquées en conseil d'administration de la SNCF, chaque produit étant dûment encarté : animaux vivants, betteraves, bière, cidre, chicorée, haricots, oléagineux, œufs, oranges, poissons de mer, pommes de terre, pommes de cidre et poires à poiré, primeurs, sucres, viandes fraîches, vin, etc.

Les transports de la marée, des fruits et légumes, du sucre ont fait l'objet de nombreux articles. Les revues à dépouiller appartiennent à la rubrique 98 "Agriculture. Transports agricoles » du plan de classement du Centre de documentation. Celles internes à l'entreprise sont particulièrement intéressantes, à l'exemple de La Revue générale des chemins de fer (désormais : RGCF), de La Vie $d u$ rail, mais aussi et surtout des revues spécifiques à la fonction commercial marchandises : Notre Trafic, Les Infos commerciales marchandises SNCF, devenues aujourd'hui Les Infos Fret.

\section{Le combat pour limiter les pertes et les avaries}

Pour assurer la bonne conservation des produits, pour la sécurité du transport, mais aussi pour réduire les coûts, le chemin de fer a toujours accordé une grande importance aux emballages et à l'amélioration de la manutention.

Plusieurs revues ont suivi la question: Emballages et leur transport, Techniques d'emballages, Transports, Courrier de la normalisation, CEGOS, Industries des plastiques modernes, Bulletin de l'AICCF', Hommes et techniques, Notre Trafic.

\footnotetext{
3- «SNCF. Les raisins de table français : variétés et principales régions productrices », brochure BC 397 ; « SNCF. Traitement rationnel des poiriers et des pommiers par M. A. Paillot », brochure BC 398, 1938.

4- «Les colis agricoles voyagent rapidement et sont livrés chez vous par la SNCF », brochure BC 441 ; « Services 1938-1939. Horaires de transports fruits et légumes des régions de production aux centres de consommations », brochure BC 396.

5- Association internationale du congrès des chemins de fer.
} 
Des manifestations ont été organisées en gare autour des techniques d'emballage, de manutention, d'arrimage. Ainsi, L'Usine se fait l'écho en 1938 des démonstrations en gare de Paris-Batignolles visant à montrer les progrès réalisés par les chemins de fer afin de supprimer les manutentions et de diminuer les coûts d'emballage et les risques d'avaries. Le Centre possède des brochures et des affiches commerciales de grand et de petit format qui incitent les expéditeurs à opter pour un emballage normalisé adapté au produit ou encore à les marquer correctement ${ }^{6}$.

Des laboratoires de recherche et d'essais sur les emballages ont vu le jour en Europe comme, en France, le Laboratoire général pour l'emballage, créé à Paris par la SNCF associée aux fabricants de la filière ${ }^{7}$. Les expéditeurs sont invités par voie d'affiches à venir y tester la qualité de leurs emballages ${ }^{8}$ (fig. 1). Pour les emballages spéciaux conçus pour la conservation des denrées périssables, le laboratoire propose une formule de cahier des charges particulier?.

La prévention des avaries reposait aussi sur une manutention correcte des marchandises et sur un arrimage efficace. Le chercheur intéressé par cette thématique pourra utilement consulter les revues Hommes et techniques, La Technique moderne, Manutention (puis Manutention stockage), Containers, Notre Trafic, La Vie du rail, la RGCF, qui ont consacré de nombreux articles aux progrès des techniques de manutention vers 1936-1937 et aux fréquents congrès de la manutention.

Par ailleurs, les Chemins de fer de l'État disposaient d'un service de prévention des avaries aux marchandises dont les actions sont notamment présentées, photographies de ses membres à l'appui, dans un article de la revue Emballages ${ }^{10}$.

Malgré tous les efforts des compagnies puis de la SNCF pour lutter contre les pertes et les avaries, les sources témoignent de l'importance des indemnités

\footnotetext{
6- AFF K 163, « Pour vos exportations de fruits et de légumes, essayez les emballages rectangulaires », 1949 ; AFF L 239, «Expédiez les œufs dans une caisse en bois », 1952 ; AFF L 330, «Utiliser les caisses marées normalisées estampillées SNCF », 1952 ; AFF L 338, « Bon emballage $=$ bon transport $»$.

7- En 1949, la SNCF et les fabricants d'emballages membres de la Société technique des industries de l'emballage ouvrent dans la gare désaffectée de Courcelles-Ceinture un premier laboratoire visant spécialement l'amélioration de l'emballage d'expédition. Il donne naissance en 1955 au Laboratoire général pour emballages installé dans un bâtiment dépendant de la gare d'Auteuil. Ce dernier travaille alors en étroite collaboration avec les laboratoires similaires de Bruxelles, Delft, Rome et Londres [N.d.l.R.].

8- AFF K 160, «Éprouvez la qualité de vos emballages, consultez le laboratoire général pour emballages ».

9- AFF L 340, 1957.

10- $\mathrm{N}^{\circ} 60$ (avril 1937).
} 

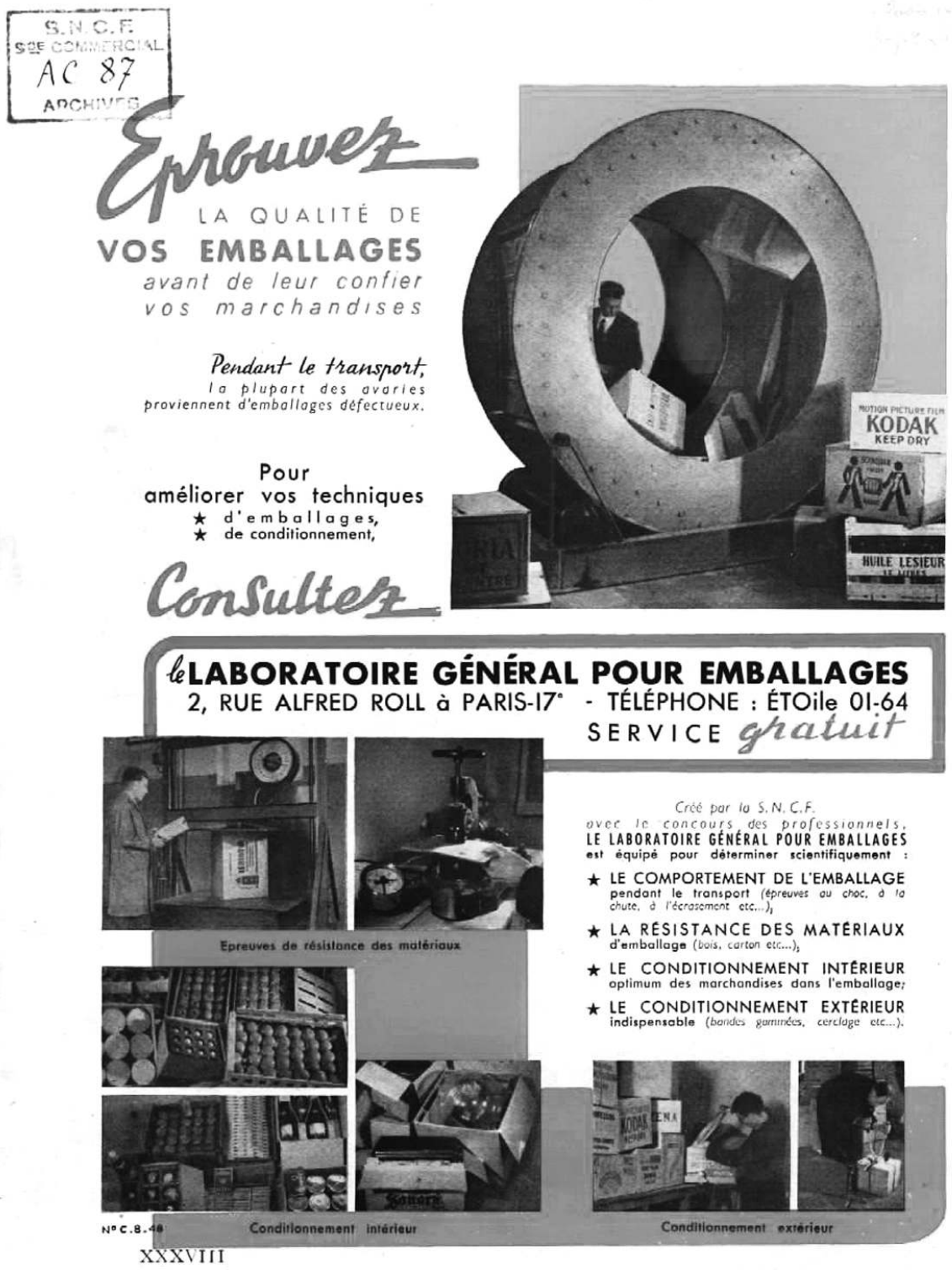

- Figure 1. CAH SNCF, cote AFF K 160. Affiche commerciale estampillée AC 87 «Éprouvez la qualité de vos emballages ... Consultez le Laboratoire général pour emballages». 
versées pour ces motifs. Citons, notamment, les dossiers documentaires du Secrétariat du conseil d'administration rassemblés sous la rubrique 9223 "Indemnités pour pertes, retards, avaries ", ainsi que bien évidemment la jurisprudence du chemin de fer. Sur le transport des denrées, celle-ci peut en effet s'avérer très intéressante à étudier. Le Centre du Mans conserve un "Relevé de la jurisprudence du chemin de fer publié entre 1933 et 1948 »" Le répertoire que nous avons réalisé en facilite l'accès par thème, de même que les index présents dans le « Relevé ». Une approche par catégorie, telle que les animaux, les fûts, les chargements et les déchargements, est ainsi possible.

\section{Le ravitaillement de la population}

Le chemin de fer a joué bien évidemment un rôle essentiel dans le ravitaillement de la population. Cela semble évident en période de guerre. Nous trouvons, au sein des archives du conseil d'administration, dans le versement 505LM, quelques dossiers sur l'organisation du ravitaillement pendant la Deuxième Guerre mondiale, ainsi que des dossiers sur le ravitaillement des cheminots pendant cette même période. À travers les demandes de consultation des cheminots adressées au Bureau des études du Service contentieux de la SNCF entre 1939 et $1943^{12}$, nous constatons l'importance vitale du jardin cheminot dans l'alimentation. Sur la thématique " cheminots et alimentation ", il faudrait aussi songer aux archives relatives aux économats, déjà bien fournies au niveau du conseil d'administration.

En dehors des périodes de guerre, la question primordiale du ravitaillement de la population transparaît dans le développement, en province et à Paris, des gares-marchés ou marchés-gares directement reliés à la voie ferrée. L'initiative remonte pourtant à 1940 , date de la première des nombreuses fiches du versement 44LM du Secrétariat du conseil d'administration consacrées aux "Gares-marchés » ${ }^{13}$ qui renvoie aux séances du conseil d'administration et à la correspondance avec le ministère des Travaux publics.

Les principales sources sont:

- pour les marchés-gares de Toulouse, Strasbourg, Nantes-État, Rouen et Saint-Étienne, les rubriques 5215 des dossiers documentaires du Secrétariat du conseil d'administration contenus dans le versement 505LM ;

11- Cote 412LM.

12- Versements 388LM et 390LM.

13- Cote archives 44LM89. 
- pour les marchés-gares de Nantes, Saint-Pol-de-Léon, Rouen, Toulouse, Rungis, Lyon, Avignon, Nice-Saint-Augustin, Marseille et Cavaillon, les boîtes d'archives 232LM 33 et 34 de la division équipements du transport, rattachée à la direction du Transport.

Pour les articles sur les gares-marchés en France et à l'étranger, le chercheur consultera les fiches d'analyses documentaires de la rubrique 637 " gares et chantiers spécialisés : gares-marchés, etc ».

M. Marois aborde, dans l'Année ferroviaire $1952^{14}$, la question primordiale du ravitaillement des grandes villes de plusieurs milliers d'habitants et notamment le problème des Halles centrales de Paris. Celles-ci traitent, en 1950, cinquante fois plus de marchandises qu'en 1850 ; elles n'ont pas de liaison avec les voies ferrées. Il expose le principe de la gare-marché reliée directement à la voie ferrée, qui permet, en supprimant les problèmes terminaux, une mise à disposition plus rapide des marchandises, un prix de revient plus bas et de meilleures conditions de vente.

À Rungis, c'est la gare-marché de Rungis, puis le Marché d'intérêt national (MIN) qui sont mis en place. Les boîtes d'archives de la division du Transport de la Région Paris-Sud-Ouest, cotées 208LM 21, 22 et 23, traitent du marché-gare de Rungis entre 1959 et 1971. Beaucoup d'articles de revues se rapportent à Rungis, premier marché mondial de denrées périssables, depuis sa construction en 1969. Des articles sont consacrés à d'autres MIN : Rouen, Nantes, et Lille.

\section{Les installations frigorifiques}

Une autre problématique centrale des compagnies de chemin de fer, puis de la SNCF, a été d'assurer la conservation des denrées périssables par le biais d'installations frigorifiques : entrepôts frigorifiques, gares frigorifiques et wagons spéciaux.

Une brochure commerciale du $\operatorname{PLM}^{15}$ (fig. 2) fait ainsi écho à un article de Sauvajol consacré à " L'équipement frigorifique du réseau PLM » publié en 1937 par la revue Transports.

Parmi les archives du Service voie et bâtiments de la Région Sud-Ouest, dans les versements 40LM et 476LM, figurent les dossiers de la construction en 1919-1920 de la gare frigorifique de Paris-Ivry (PO). Sur cette même gare, le centre possède aussi un ouvrage coté 63J2.

14- Cote fonds documentaire 030L48.

15- Dans le versement 470LM, la brochure « Des denrées fraîches grâce à l'équipement frigorifique réalisé sur le PLM », BCP 320, 1937. 

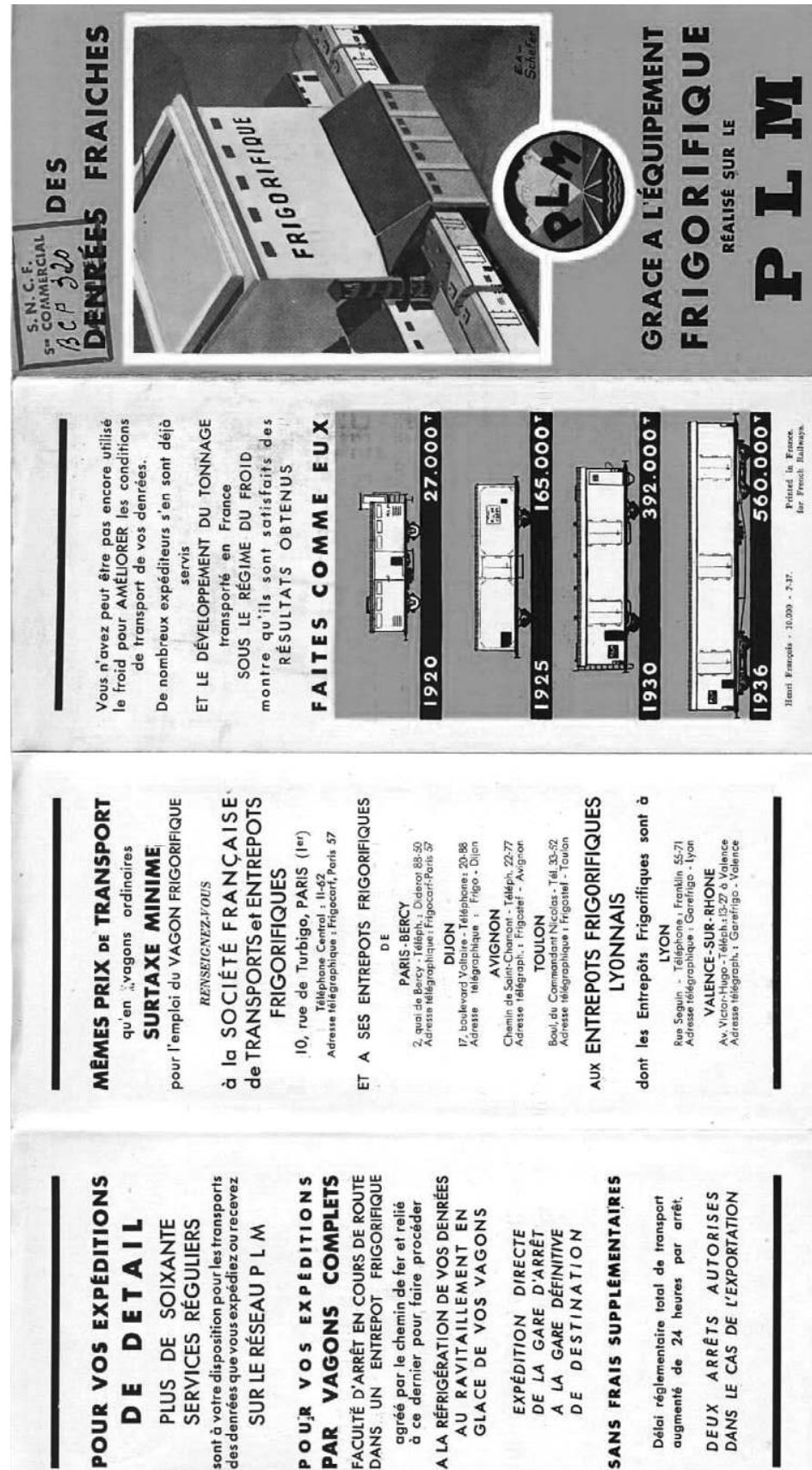

- Figure 2. CAH SNCF, cote 470LM12. Brochure commerciale du Paris-LyonMéditerranée estampillée BCP 320 « Des denrées fraîches grâce à l'équipement frigorifique réalisé sur le PLM ». 1937. 
Dans notre fonds d'ouvrages et de revues hérité du Centre de documentation, le chercheur pourra consulter les rubriques 637 " Gares et chantiers spécialisés - dont gares frigorifiques » et 975 «Installations frigorifiques. Entrepôts frigorifiques. Stations de reglaçage ». Les revues à dépouiller sont essentiellement : le Bulletin de l'Institut national du froid, la Revue générale du froid, la revue Transports, La Vie du rail et de nombreuses revues étrangères. Citons, à titre d'exemple, l'article de Duchesnoy, paru en juillet 1933 dans Les Chemins de fer et les Tramways, consacré aux entrepôts frigorifiques et gares laitières. Il y évoque les installations du réseau de l'État, notamment la gare laitière de Vouillé, l'entrepôt frigorifique et la gare de Vaugirard-marchandises, et celles de la Compagnie d'Orléans à Chevaleret.

La RGCF et La Vie du Rail consacrent de leur côté plusieurs articles à la gare frigorifique de Perpignan, notamment en 1944 (description des ouvrages en béton armé) et en 1957 (évocation de la concurrence des entrepôts frigorifiques). D'autres installations frigorifiques sont évoquées dans les revues, telles celles de Vitry entre 1962 et 1973, de Saint-Brieuc.

Il ne faut pas oublier le thème des relations entre la SNCF et les sociétés exploitant des entrepôts frigorifiques, à l'exemple de la Société de transports et d'entrepôts frigorifiques (STEF), sa filiale. Les dossiers du Secrétariat de la direction générale présents sous les cotes 68LM352, 68LM353 et 20LM896 traitent de ce sujet.

Toujours dans le versement 20LM, un dossier de la boîte 20LM896 contient une étude intéressante sur l'équipement frigorifique des régions SNCF Ouest et Sud-Ouest en lien avec le plan d'équipement national du ministère de l'Agriculture.

\section{Le matériel de transport}

Il était important pour le chemin de fer de disposer d'un matériel adapté aux besoins particuliers, de chercher à augmenter la charge transportée, sans oublier les obligations de normalisation des wagons fixées par l'Union internationale des chemins de fer à partir de 1922.

Sur le matériel à température dirigée - wagons calorifiques, wagons isothermes, réfrigérants ou frigorifiques - pour le transport des denrées périssables, les revues à dépouiller sont le Bulletin de l'Institut national du froid, la Revue générale du froid, le Bulletin de l'UIC et la RGCF pour des articles tant de l'époque des compagnies que de la SNCF.

Ainsi, les essais de nouveaux procédés de calorifugeage pour wagons isothermes du PO ont donné lieu à des articles en 1933 dans la $R G C F$ et le Génie 
civil. Cette même compagnie a publié un ouvrage sur ses wagons isothermes et voitures réfrigérées mis en avant lors de l'exposition coloniale de $1931^{16}$.

Les congrès internationaux sur le thème ont donné lieu à de nombreux articles, ainsi que l'évolution du glaçage des wagons réfrigérants en 1953. J.-B. Verlot, ingénieur à la Direction commerciale de la SNCF, est quant à lui l'auteur de plusieurs articles dans la Revue générale du froid dans les années 1960.

De manière plus générale, les rubriques documentaires à consulter sont les rubriques 44 et 45 du plan de classement du Centre de documentation de la SNCF.

Du côté des archives, nous trouvons surtout des sources sur la construction et l'acquisition de wagons. C'est précisément l'intitulé de la rubrique 2223 du plan de classement des dossiers documentaires du Secrétariat du conseil d'administration présents dans le versement 505LM. Un autre versement intéressant est le 43LM qui contient de très nombreux dossiers de marchés pour la construction ou l'acquisition de wagons, wagons primeurs, wagons réfrigérants, wagons spéciaux, etc. Plus anecdotiques, des brochures publicitaires, publiées régulièrement par la direction commerciale de la SNCF, présentent le parc de wagons de marchandises ${ }^{17}$.

À signaler aussi, dans le versement 68LM du Secrétariat de la direction générale, des dossiers sur les participations financières de la SNCF à la société internationale Interfrigo.

\section{Palettes, conteneurs, mécanisation de la manutention et mécanisation des halles}

La volonté d'améliorer les conditions de chargement et de déchargement et de réduire ainsi les coûts de manutention a conduit au développement de l'utilisation de la palette, puis du container, et à la mécanisation de la manutention.

Au sujet des palettes, le chercheur pourra consulter la rubrique $692.4 \mathrm{du}$ plan de classement du Centre de documentation.

Des articles parus dans les revues étrangères relatent le mouvement de palettisation dans le trafic de détail aux États-Unis dans les années 1948-1949, ainsi que le phénomène de normalisation de la dimension des palettes, tout en

16- Cote fonds documentaire 13J 8. «Compagnie de Paris-Orléans. Exposition coloniale de 1931. Wagons isothermes, voitures réfrigérées. »

17- Cote fonds documentaire 580VR11 « Répertoire général du matériel roulant marchandises », 1962, et son complément, en 1979, «Des wagons pour vos marchandises » sous la cote 580VR9. 
mettant en avant les problèmes posés par la palettisation pour les transports internationaux.

Côté français, les revues à dépouiller sont la revue Emballages, Courrier de la normalisation, le Bulletin documentaire de la $S C E T A^{18}$, ou encore la revue interne à la SNCF Notre Trafic.

La palette, palette plate et palette-caisse, s'est répandue à la SNCF et chez les chargeurs. Les brochures commerciales présentaient les avantages tarifaires accordés aux utilisateurs de "palettes agréées ». Un nouveau tarif, le $\mathrm{n}^{\circ} 114$, concernant le transport de marchandises chargées sur palettes agréées, a été institué en 1951.

Beaucoup de fiches d'analyses documentaires évoquent le pool international des palettes pour le trafic des denrées, créé en 1961 entre les administrations des chemins de fer allemands, autrichiens, belges, français, italiens, luxembourgeois, néerlandais et suisses. Le système est le suivant : les palettes sont louées au chemin de fer, cinquante-six palettes forment le chargement d'un wagon spécial et il existe un accord entre les membres pour le remplacement ou la réparation des palettes, l'Office central du transport des denrées assurant le contrôle du mouvement des wagons.

Paul-Émile Javary, directeur de l'Exploitation de la Compagnie du Nord, a publié dans la $R G C F$, en 1929, 1930 et 1933, plusieurs articles traitant de l'emploi des " cadres " dans le transport des marchandises. En 1934, les containers appartiennent soit aux réseaux de chemins de fer, soit à leurs clients. En 1936, une nouveauté technique apparaît avec l'abandon du type à galets au profit de celui à boucles à levage, mais des perfectionnements sont encore nécessaires.

Toutes les références documentaires se trouvent à la rubrique $692 \mathrm{du}$ plan de classement du Centre de documentation et aux sous-rubriques suivantes : 692.1 pour les containers ordinaires, 692.2 pour les cadres isothermes ou réfrigérants, spécifiques aux denrées périssables, 692.3 pour les réservoirs à liquides tel la bière, le vin et l'huile, et 692.4 pour les palettes pour les colis.

Les revues traitant du sujet sont, essentiellement, le Bureau international des containers, le Bulletin de l'UIC, les revues Le Container, CEGOS, Hommes et techniques, la $R G C F$ et Manutention. Pour les cadres isothermes ou réfrigérants, il s'agit surtout du Bulletin international du froid et des revues étrangères.

18- Société de contrôle et d'exploitation des transports auxiliaires, fondée en 1942. Elle regroupe initialement les filiales automobiles issues des grands réseaux [Stplm (PLM), Starn (Nord), Sate (Est), Stapo (PO), Satam (Midi), Satos (État)] et les bureaux de ville voyageurs et marchandises de Paris [N.d.l.R.]. 
De nombreux articles sont consacrés aux congrès internationaux des containers, ainsi qu'aux expositions internationales, ou encore, en 1954, à la Compagnie nouvelle des cadres (CNC), filiale de la SNCF créée en 1948, et à son rôle dans le développement de la conteneurisation en France.

La mécanisation de la manutention lors des opérations de chargement et de déchargement qui se déroulent chez ses clients est une préoccupation du chemin de fer.

Ainsi, la SNCF aide sa clientèle à moderniser sa manutention par le biais de manifestations comme le train de la manutention SNCF, par des présentations de matériel de manutention aux salons de la manutention, ou encore par des affiches.

Signalons aussi le rôle de la Société auxiliaire de manutention moderne (SAUMMO), filiale SNCF créée en 1956, qui fournit aux clients les appareils dont ils peuvent avoir besoin et procède à des études d'organisation intérieure des manutentions. La SAUMMO travaille étroitement avec le Service manutention-transport de la Direction commerciale de la SNCF ${ }^{19}$.

Le thème est abordé dans les fiches d'analyses documentaires de la rubrique 638 du plan de classement du Centre de documentation intitulée « transport des marchandises par voie ferrée, organisation de la manutention des colis, mécanisation de la manutention ». La revue Le Bulletin de documentation de la SCETA évoque les engins de manutention (transpalettes, élévateurs) utilisés pour le camionnage de la SNCF.

L'amélioration des opérations de manutention, par le biais de la mécanisation, concerne bien évidemment les halles des chemins de fer.

En 1948, une nouvelle organisation du transport des colis de détail est mise en place. La modernisation des halles de détail à la SNCF s'inspirait de l'exemple anglais. Les gares habilitées aux expéditions de détail ont été équipées pour la manutention des palettes et des palettes-caisses. Des chantiers séparés ont été mis en place et l'organisation du travail a été revue.

Un ouvrage, en date de 1947, de la Commission du trafic terminal ${ }^{20}$, traite de la modernisation des halles de détail à la $\mathrm{SNCF}^{21}$, modernisation qui génère une augmentation du rendement. La halle mécanisée de Paris-Tolbiac a

19- Créé en 1951 au sein de la Direction commerciale de la SNCF, le « Service manutentiontransport (et engins de porte à porte) » avait pour objet d'apporter aide et conseil dans les études d'organisation des manutentions terminales [N.d.l.R.].

20- Le but principal de cette commission SNCF est d'examiner tous les travaux des régions qui ont trait à l'organisation du trafic terminal de détail dans Paris et sa banlieue et dans certaines grandes agglomérations de province.

21- Cote 610VR9. 
ainsi inspiré un ouvrage ${ }^{22}$ et plusieurs articles. En avril 1959, la $R G C F$ consacre un article à la palettisation d'un grand centre d'expédition de détail : la gare de Lille Saint-Sauveur.

\section{Les embranchements particuliers}

Détaillant la chaîne du producteur au consommateur, il nous faut consacrer un paragraphe aux embranchements particuliers et à l'installation, dans les emprises du chemin de fer, de silos, de laiteries, de coopératives agricoles.

Le Centre des archives historiques conserve des archives de la direction du Transport sur les embranchements particuliers. Toutefois l'analyse actuelle des dossiers ne permet pas de savoir s'il s'agit d'embranchements agricoles ou industriels. Nous possédons également les archives de la division commerciale fret de la direction régionale de $\operatorname{Lyon}^{23}$, celles de la direction régionale de Rennes ${ }^{24}$, et nous avons une prévision de versement venant de la direction régionale de Dijon. Dans ces fonds nous trouvons les conventions d'occupation relatives à des embranchements particuliers agricoles mais aussi des occupations en gare. Les dossiers traitent aussi des modifications des installations et des déposes d'embranchements.

Côté fonds documentaire, la question des embranchements particuliers est évoquée dans différentes rubriques du plan de classement, selon l'angle commercial ou l'angle technique. À titre d'exemple, citons l'article, paru dans la $R G C F$ en octobre 1967 , relatif à la construction et à la desserte ferroviaire du nouvel atelier d'embouteillage de la Société des eaux d'Évian.

\section{Le "problème terminal "}

Face à la concurrence, le chemin de fer se devait de réduire les frais afférents aux transports terminaux : coût des opérations de camionnage et de transbordement. Roger Guibert, alors directeur général de la SCETA, a tenu plusieurs conférences sur le sujet, notamment à Londres en 1950 : «Et le chemin de fer vivra : quelques réflexions sur l'avenir des chemins de fer français et le problème terminal. " Il convient donc d'examiner les sources à disposition sur les solutions apportées par le chemin de fer en ce domaine, à savoir sur les " garescentres ", les agences, "les gares-îlots ", les services de factage et le camionnage.

22- Région SNCF Sud-Ouest. Paris-Tolbiac : notice sur le chantier de manutention, 1952, 22 p., ouvrage coté 610VR8.

23- Versement 300LM.

24- Versement 506LM. 
La mise en place, le $1^{\text {er }}$ janvier 1946 , du "régime accéléré " (RA) ${ }^{25}$, consenti d'office, sans augmentation de la taxe normale de transport, aux marchandises qui nécessitaient un acheminement rapide (dont les animaux vivants et denrées périssables), conduit à la mise en place progressive d'une centaine de "gares-centres ». Placées au centre d'importantes zones de trafic et dotées des installations de triage et de transbordements spéciales au RA, ces gares étaient destinées à réduire au maximum les escales intermédiaires. Elles échangeaient entre elles, par trains accélérés, des wagons complets de détail.

Cette notion de gare-centre est étendue, au début des années 1950, aux gares qui, dans le cadre de la coordination et de l'harmonisation des transports ferroviaires et routiers (articles 25 et 26 du décret du 14 décembre 1949), ont été appelées à compenser les conséquences de la substitution de dessertes routières aux lignes secondaires dont le faible trafic ne justifiait plus le maintien. L'exemple d'Angoulême est exposé dans la $R G C F$ en 1953 : menée en 1951, l'opération a conduit à la fermeture de $241 \mathrm{~km}$ de lignes et à la concentration du trafic sur cinq gares-centres ${ }^{26}$. On notera que ces gares, comme pour celles des expériences similaires poursuivies cette même année dans les régions d'Angers et de Chalon-sur-Saône, étaient rattachées à un chef d'agence qui regroupait entre ses mains les fonctions de mouvement et les fonctions commerciales autrefois assurées par l'inspecteur du Mouvement et le chef de la circonscription commerciale. Cet agent devenait ainsi le représentant local unique de la SNCF. M. Bidet, dans la revue interne à la SNCF Notre Trafic de novembre 1954, consacrée aux agences et aux garescentres de la SNCF, expose l'intérêt du système de desserte routière pour les transports terminaux. Le chemin de fer, écrit-il, conserve la responsabilité du transport de bout en bout et demeure en contact direct avec la clientèle.

En banlieue parisienne, les " gares-îlots » étaient un exemple de modernisation des services terminaux. La nouvelle organisation de livraison et d'enlèvement à domicile des colis dans la banlieue parisienne reposait en effet sur

25- Jusqu'en 1946, l'acheminement des marchandises relevait des régimes de la grande et de la petite vitesse (GV et PV), le premier se différenciant du second par des délais de transport plus courts mais des tarifs plus élevés. Ce système excluait de la GV toutes les marchandises de peu de valeur, y compris celles dont la nature (comme les produits agricoles) exigeait un transport rapide. À l'inverse, les régimes accéléré et ordinaire (RA et RO) tenaient compte davantage de la nature des marchandises que de leur valeur, celles expédiées en RA bénéficiant désormais de délais équivalents à l'ancienne GV sans être financièrement pénalisées. On signalera cependant la création en 1935 de tarifs à vitesse unique associés à des trains spécialisés à acheminement accéléré. Appliquée dans un premier temps au colis de moins de $50 \mathrm{~kg}$, puis au transport des animaux vivants, cette solution fut étendue le $1^{\text {er }}$ août 1944 au trafic des denrées périssables [N.d.l.R.].

26- $\mathrm{N}^{\circ} 4$ (avril 1953). 
la création de vingt gares destinées à servir de relais entre les grandes gares où ceux-ci étaient réceptionnés et leurs destinataires. Le transfert depuis les gares terminus jusqu'aux gares-îlots était assuré par des véhicules lourds (remorques tirées par des tracteurs Diesel Panhard et Latil) et au-delà, dans un rayon de 6 à 7 km, par des camions. Fin 1948, dix gares-îlots étaient en service en région parisienne. Les articles consacrés aux gares-îlots de la banlieue parisienne sont nombreux. Signalons aussi l'ouvrage, coté $25 \mathrm{~K} 3$, intitulé Les Îlots de livraison des services de factage SNCF à Lyon et à Paris.

Les remorques rail-route étaient également une solution adoptée pour résoudre le problème des équipements terminaux.

Les bureaux de ville étaient à la fois centres des enlèvements à domicile, bureaux-marchandises, bureaux-voyageurs et centres des renseignements téléphonés. Nombreux sont les articles sur les bureaux de ville parisiens en 1954 qui traitent de leur rôle, de leur organisation, de leur collaboration avec la SCETA, et évoquent Orsay, le centre de tri des bureaux en question.

Sur le factage et le camionnage, le Centre du Mans possède plusieurs ouvrages édités par la SCETA ${ }^{27}$. Vingt-quatre fiches du versement $44 \mathrm{LM}$ concernent le camionnage et le factage entre 1938 et 1958, dont douze sur Paris, et renvoient aux décisions du conseil d'administration sur le sujet. Il en est de même pour les dossiers du versement 505LM des rubriques suivantes : rubrique 5211 "services de correspondance : traités, tarification, contrôle ", rubrique 5212 «bureaux de ville».

\section{Restauration ferroviaire}

La restauration ferroviaire - buffets et wagons-restaurants - est le dernier aspect de notre exposé.

La thématique des buffets est illustrée par une affiche de 1951 vantant les buffets gastronomiques ${ }^{28}$, document repris par La Vie du rail en $1984^{29}$ dans l'article de Michel Chlastacz, "Les buffets de gare : 150 ans de gastronomie ferroviaire ", sujet qui, quelques mois plus tôt, avait également inspiré le Bulletin d'information des cadres ${ }^{30}$.

27- Ouvrages sous la rubrique $25 \mathrm{~K}$ du Centre de documentation de la SNCF.

28- VDR 317.

29- No 1942 (3 mai 1984).

30- $\mathrm{N}^{\circ} 128$ (janvier-avril 1984). 
Côté archives, les sources relatives aux buffets sont nombreuses et diversifiées :

- décisions du conseil d'administration de la SNCF de 1938 à 1959, dont l'accès est facilité par les neuf fiches du versement 44LM relatives au sujet ${ }^{31}$;

- dossiers documentaires du secrétariat du conseil d'administration (505LM), notamment les dossiers de la rubrique 534 "buffets et buvettes", qui traitent du contrôle et de la surveillance exercés par la SNCF sur les dits établissements, et ceux de la rubrique 9223 " traités pour l'exploitation de buffets ", qui se rapportent aux concessions. N'étant pas propriétaires de leur fond, les buffetiers étaient soumis à un cahier des charges (rubrique 2919 "Cahier des charges du service des buffets », 1946);

- dossiers du secrétariat de la direction générale ;

- études de marché de la direction commerciale Voyageurs (649LM) telles celles de 1977 (649LM183) ou encore de 1988 ( « Les buffets de gare et leur clientèle », 649LM185).

Parmi les autres fonds conservés par le Centre des archives historiques du Mans, citons aussi les archives des services Voie et Bâtiments des Compagnies de l'Est, du PO-Midi et du Nord qui donnent des indications relatives aux travaux de construction et d'aménagements des buffets.

Enfin, au nombre des pièces versées par la SNCF aux Archives Nationales dans les années 1960, nous trouvons, aux Archives nationales du monde du travail à Roubaix, des dossiers sur les buffets hérités du Service Exploitation de la Compagnie du Nord (202AQ C710, période 1926-1947).

Longtemps limitée aux seuls buffets, la restauration s'invite à bord des trains en 1882, année de l'inauguration par la Compagnie des wagons-lits de son premier wagon-restaurant entre Marseille et Nice. Sur ce thème, les archives de la SNCF conservent des versements similaires à ceux se rapportant aux buffets :

- décisions du conseil d'administration de la SNCF traitant surtout de la question de la participation de la SNCF dans les recettes de la CIWL, accessibles par les cinq fiches du versement 44LM consacrées aux « wagons-lits restaurants $\aleph^{32}$;

- dossiers documentaires du secrétariat du conseil d'administration (505LM), rubriques 5320 et 5321 relatives aux contrats conclus pour la restauration (notamment entre la SNCF et la $\mathrm{CIWL}^{33}$ ) et à leur exécution ;

31- Cote archives 44LM82.

32- Cote archives 44LM105.

33- Sur les relations SNCF-CIWL, voir aussi 68 LM. 
- dossiers du secrétariat de la direction générale (dont ceux de Paul Gentil, directeur général adjoint puis directeur général) sur la restauration entre 1972 et 1984 et d'autres encore sur les années 1983-1984 ;

- études de marché de la direction commerciale Voyageurs puis de la direction Grandes Lignes (649LM) sur la restauration entre 1977 et 2004 : enquêtes, tests sur certaines relations ("Test de restauration sur Paris-Lille en 1978 ", 649LM23), baromètres de satisfaction de la clientèle (" Baromètre restauration 1993 ", 649LM289);

- dossiers de la division du trafic-voyageurs (versement 252LM de la direction commerciale), notamment les rubriques 528-104 sur la révision des conventions entre la CIWL et les réseaux européens, 528-150 sur les wagons-

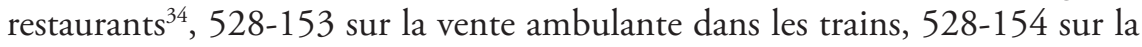
restauration dans les « rames grand parcours ", 528-160 relatifs au groupe de travail SNCF/CIWL sur la restauration de 1954 à 1974, ou 528-300 sur la restauration par d'autres sociétés que la CIWL.

Un versement de la direction Voyages France Europe est prévu. Il comprend une trentaine de boîtes d'archives du département Produits de la direction Grandes Lignes sur la restauration ferroviaire dans les années 1990 à 2001.

Nous ne pouvons terminer le sujet sans évoquer les documents iconographiques en notre possession, au premier rang desquels les affiches. Citons notamment l'affiche de la Compagnie du Nord et de la CIWL «De nouveaux wagons-restaurants " ${ }^{35}$, ou encore celle de Fix-Masseau, "Wagonsrestaurants ", de $1981^{36}$. Nous conservons également, dans le versement 490 LM de la direction Grandes Lignes, des photographies de restauration à bord sous forme d'affichettes datant des années 1970. Les collections de la CIWL méritent également d'être consultées.

\section{Conclusion}

Nous espérons vous avoir convaincu de l'intérêt de venir au Mans exploiter nos sources. Le Centre est ouvert du lundi au vendredi de 8 h 15 à $12 \mathrm{~h}$ et de $13 \mathrm{~h}$ à $17 \mathrm{~h}$. Actuellement, l'accès à la salle de lecture se fait sur rendez-vous ${ }^{37}$, essentiellement parce que nous préparons la venue des lecteurs car l'usage de nos bases de données, tant en archives qu'en documentation, est

34- 74 dossiers, datant de 1938 à 1973, de la cote 252LM96 à la cote 252LM102.

35- Cote affiche VDR 1033.

36- Cote affiche VDR 2039.

37- Par mèl à l'adresse suivante : archiveshistoriques.sncf@sncf.fr ou en téléphonant au 0243 217465. 
réservé au personnel du centre. Cette situation devrait bientôt évoluer suite à l'acquisition d'un nouveau logiciel Archives. Une réflexion est en cours également pour les fonds documentaires de notre Centre et ceux du Centre de documentation situé à Paris, 45 rue de Londres.

Pour en revenir à notre thème d'étude, notre Centre d'archives présente bien sûr encore des lacunes : insuffisance des sources produites par la SNCF provenant du service commercial puis de la direction commerciale, de la direction du Mouvement puis de la direction du Transport, et des services Exploitation des régions. Le constat vaut aussi pour l'époque des compagnies. Toutefois, vous l'aurez compris, il convient d'associer les sources archivistiques présentées aujourd'hui à la grande richesse de nos fonds documentaires. 